\title{
Frecuencia y factores asociados a episiotomía en primigestas atendidas en el Hospital "Luis F. Martínez".
}

(c)(1)(9)

Frequency and factors associated with episiotomy in primigravidas attended at "Luis F. Martinez."

MD. Christian Moncayo Rivera. ${ }^{1} \&$ MD. Viviana Vázquez Becerra. ${ }^{2}$

Recibido: 08-03-2017 / Revisado: 08-05-2017 Aceptado: 13-06-2018/ Publicado: 01-07-2018

\begin{abstract}
.
DOI: https://doi.org/10.33262/cienciadigital.v2i3.151

The research aims to determine the risk factors associated with episiotomy in primitive women treated at the Hospital "Luis F. Martínez", during the year 2016. It is a documentary, descriptive, retrospective, cross-sectional study. field with the collection of data from medical records, based on the inclusion criteria derived from the objectives. Charts and graphs were developed using Microsoft Excel 2016 and the SPSS program, version 20. Among the results obtained, it was found that the number of eutocic or normal deliveries in 2016 was 453, of which 300 (66.22\%) are multiparous and $153(33.77 \%)$ are nulliparous, the type of episiotomy performed was $100 \%$ lateral half of the cases. The prevalence of episiotomy in primiparous patients in the year of study was $64.05 \%$, a value higher than that recommended by the WHO and others obtained in similar regional investigations. Among the risk factors found associated with episiotomy, we have: cephalic perimeter, weight and height of the newborn, age fulfilled and position of lithotomy of the mother at the time of delivery; being gestational age of higher term a protection factor.
\end{abstract}

Keywords: Prevalence, Risk Factors, Episiotomy, Primigraves

${ }^{1}$ Hospital “Luis F. Martínez", Cuenca,Ecuador, cmoncayo@gmail.com

${ }^{2}$ Hospital "Luis F. Martínez", Cuenca,Ecuador, cmoncayo@gmail.com 


\section{Resumen.}

La investigación pretendió determinar la frecuencia y Factores asociados a la episiotomía en mujeres primigestas atendidas en el Hospital "Luis F. Martínez", durante el año 2016. Se trata de un estudio documental, descriptivo, retrospectivo, de tipo transversal Se inicia la investigación de campo con la recolección de datos de las historias clínicas, en base a los criterios de inclusión derivados de los objetivos. Se elaboraron tablas y gráficos utilizó Microsoft Excel 2016 y el programa SPSS, versión 20. Entre los resultados obtenidos se encontró que el número de partos eutócicos o normales en el año 2016 fue de 453, de las cuales 300 (66.22\%) son multíparas y 153 (33.77\%) son nulíparas, el tipo de episiotomía realizada fue medio lateral el 100\% de los casos. La prevalencia de episiotomía en pacientes primigestas en el año de estudio fue de $64.05 \%$, valor superior al recomendado por la OMS y a otros obtenidos en investigaciones regionales semejantes. Entre los factores de riesgo encontrados asociados a la episiotomía, tenemos: perímetro cefálico, peso y talla del neonato, edad cumplida y posición de litotomía de la madre al momento del parto; siendo la edad gestacional de termino superior un factor de protección.

Palabras Claves: Prevalencia, Factores de Riesgo, Episiotomía, Primigestas.

\section{Introducción.}

La episiotomía se define como una incisión en el periné para facilitar el parto vagina. Según la OMS, se trata de un procedimiento quirúrgico menor, realizado en su mayoría, en sentido medio lateral o en la línea media del periné (1). A nivel mundial el uso de la episiotomía actualmente está en discusión, se cuestiona si es realmente necesario realizarla, especialmente a aquellas que vivirán el parto vaginal por primera vez. Rodríguez, en España, en su estudio comparativo de incidencia de episiotomía con anestesia epidural, realizado en Madrid (España) el año 2012, manifiesta que la episiotomía es uno de los procedimientos quirúrgicos más comunes de la medicina occidental. La evidencia científica recomienda disminuir la tasa de episiotomías, además, considera que a partir de conductas oportunas y reflexivas se puede evitar la episiotomía en gran parte de las nulíparas (5). La episiotomía restrictiva tiene varias ventajas sobre la episiotomía de rutina, entre ellas: menor trauma perineal posterior, menor sutura y menos complicaciones de cicatrización. Aunque el enfoque restrictivo estuvo asociado con un mayor riesgo de traumatismo perineal anterior, en varios estudios realizados, no hubo diferencias entre los dos enfoques en el traumatismo vaginal o perineal grave o en la mayoría de las medidas de dolor (6).

Como resultado, la Organización Mundial de la Salud (OMS, 2016) recomienda que la episiotomía se realizara sólo para un número estrictamente limitado de indicaciones (5), establece un máximo de un 16-30\%(1). Aunque en la práctica ginecológica moderna no tiene ningún lugar la episiotomía sistémica, algunas indicaciones clínicas justifican su uso, como complicaciones fetales: bradicardia terminal o distocia de hombros o para controlar la 
lesión perineal, en partos vaginales instrumentados, laceración grande previa, cuerpo perineal pequeño o niño grande (2).

En Ecuador, en el año de 2008 el Ministerio de Sanidad, a través de la Normativa Materno Neonatal, elabora estrategias restrictivas de atención al parto normal, cuyo propósito fue adecuar su asistencia a una práctica basada en la evidencia científica (2). Hay factores predeterminantes para llevar a cabo este tipo de procedimiento obstétrico en un parto vaginal espontaneo: nuliparidad, perímetro cefálico, peso del recién nacido superior a $3000 \mathrm{~g}$, uso de anestesia epidural, el parto inducido, expulsivo más de una hora y el uso fórceps (3). Al utilizar maniobras apropiadas de atención al parto, el tamaño de la circunferencia fetal no fue un factor influyente en la producción de desgarros al no practicar la episiotomía (15). De igual manera en un estudio realizado en el año 2007 en el Hospital Gineco - Obstétrico Isidro Ayora de Quito, que según estadísticas analizadas se entendieron a 7.459 partos vaginales, registrándose un $42,6 \%$ de casos con episiotomía (16). En la investigación realizada por Ramírez D. titulada "Uso de Poligactina 910 2/0 Vs Catgut Crómico en episiotomía de pacientes primigestas. Hospital Vicente Corral Moscoso. Cuenca. 2010”, concluye que, de una muestra de 3.392 partos vaginales, el 11.79\% (400) eran mujeres primigestas a las que se realizó episiotomía. (17). Este procedimiento quirúrgico se debe realizar con anestesia local o regional y previo consentimiento verbal o escrito de la paciente (19)

Sin embargo, una de las principales razones por la cual se realiza la episiotomía como práctica de rutina es la prevención de desgarros perineales graves de tercer y cuarto grado debido a las complicaciones que estas conllevan como la incontinencia anal, laceraciones perineales mayores y consiguiente duración de la estancia hospitalaria (26).

En la zona de influencia del Hospital Luis F. Martínez, cantón Cañar, no se han detectado investigaciones relacionadas, ni tampoco estadísticas confiables que justifiquen el uso rutinario de la episiotomía, a pesar de existir casos derivadas por complicaciones posteriores (3).

Con estos antecedentes se inicia la investigación, con el objeto de determinar la prevalencia y factores de riesgo asociados a la episiotomía; identificar los grupos de estudio de acuerdo a las diferentes variables socio-demográficas, identificar los factores asociados como: peso, talla del recién nacido, perímetro cefálico fetal, edad gestacional y edad materna con la realización de episiotomía.

A pesar de ello, en revisiones bibliográficas se admite que, el procedimiento a elección y no de rutina arroja algunas ventajas de la Episiotomía; entre las cuales tenemos: minimiza trauma de piso pélvico, aumenta luz del canal de parto, disminuye el tiempo del periodo expulsivo, evita sufrimiento fetal. Entre las complicaciones: Posibilidad de provocarse hematoma perineal, desgarro perineal severo (III-IV), reconstrucción insatisfactoria, dolor post parto en el perineo, posible Infección, mala y tardía cicatrización. (11). Resultando interesante y necesario analizar las causas que predisponen a los profesionales a continuar 
con este tipo de procedimiento, así como encontrar alternativas que eviten el uso injustificado de la episiotomía y llevar al parto a un proceso más fisiológico, conociéndose como humanización del parto (6).

Con esta perspectiva se propone al mismo tiempo, comprobar la hipótesis de investigación relacionada con la aplicación de la Normativa Materno Neonatal del Ministerio de Salud Pública, publicada en el 2008 y vigente en el Ecuador y la disminución de la práctica de la episiotomía en pacientes primigestas en el Hospital Luis F. Martínez, disminución que se asociados directamente con la presencia de los factores de riesgo estudiados, con: perímetro cefálico fetal, peso y talla del neonato, edad gestacional de término, edad, posición al momento del parto y edad gestacional.

\section{Metodología.}

Se trata de un estudio de tipo documental, descriptivo, retrospectivo y de corte transversal, desarrollado en el servicio de Ginecología del Hospital Luis F Martínez de la Ciudad de Cañar, en mujeres primigestas que acudieron a la consulta durante el año 2016. Se inicia detectando una población de 153 mujeres con partos eutócicos y una muestra de 98 casos de primigestas que recibieron episiotomía. Se rechazan historias clínicas incompletas, eligiendo aquellas que cumplan con los criterios de inclusión.: pacientes primigestas con embarazo a término, parto eutócico o normal sin complicaciones, feto único. Usando ficha de recolección de datos se clasificaron, ordenaron e ingresaron a una hoja de Excel, para obtener la prevalencia mediante los primeros resultados de frecuencias en Tablas y Gráficos; datos que posteriormente ingresados al SPSS (herramienta informática), fueron básicos en la obtención de la Razón de Prevalencia, intervalos de confianza y chi-cuadrado de Pearson como determinante del grado de asociación entre variables; datos con los cuales se posibilitaron calificarlos como factores de riesgo. Para identificar factores de riesgo y protectores se realizó un análisis de regresión logística binomial. Por último se obtienen las conclusiones mediante una contrastación discutida entre los resultados históricos similares y los datos obtenidos en el estudio.

\section{Resultados.}

Al caracterizar la muestra en estudio mediante el análisis de los datos socio demográficos, se observa que el mayor porcentaje de mujeres atendidas $(84.97 \%, 130$ de las 153 , con o sin Episiotomía), se encuentran en el rango de edad de 15 a 25 años, en un porcentaje similar con edades entre los 14 y 36 años de edad. El 50.33\%, (77 de las 153 mujeres), se consideran de etnia indígena. El mayor porcentaje (42.48\%) viven en unión libre y con formación educativo Básica y Bachillerato, el 85.62\%.

Tabla $\mathbf{N}^{\circ}$ 1. Frecuencia de Episiotomía en mujeres primigestas. 


\begin{tabular}{ccc}
\hline Episiotomía & Frecuencia & Porcentaje \\
\hline SI & 98 & $64.05 \%$ \\
\hline NO & 55 & $35.95 \%$ \\
\hline TOTAL & 153 & $100.00 \%$ \\
\hline
\end{tabular}

Fuente: Historias clínicas del Hospital, 2016.

Elaborado por: El investigador.

En relación a la prevalencia encontrada en la muestra y durante el período de estudio, en la Tabla $\mathrm{N}^{\circ} 1$, se observa que se han realizado la episiotomía 98 de las 153 mujeres atendidas, llegando a una prevalencia de Episiotomía del 64.05\% en el Hospital "Luis F. Martínez", valor superior al establecido por la OMS.

Al analizar los factores considerados por la OMS como de riesgo para la aplicación de la episiotomía en mujeres primigestas, tales como: peso y talla del recién nacido, perímetro cefálico fetal, edad gestacional de termino y edad materna, posición de la madre al momento del parto; se encontraron las siguientes particularidades:

Tabla $\mathbf{N}^{\circ}$ 2. Relación entre la episiotomía y el perímetro cefálico fetal.

\begin{tabular}{|c|c|c|c|c|c|c|c|c|c|}
\hline \multirow{3}{*}{$\begin{array}{c}\text { PERÍMETRO } \\
\text { CEFÁLICO }\end{array}$} & \multicolumn{6}{|c|}{ EPISIOTOMÍA } & \multirow{3}{*}{$\begin{array}{l}\text { Razón de } \\
\text { Prevalencia }\end{array}$} & \multirow{3}{*}{$\begin{array}{c}\text { Intervalo de } \\
\text { Confianza }\end{array}$} & \multirow{3}{*}{$\begin{array}{l}\text { Valor } \\
\text { de } p\end{array}$} \\
\hline & \multicolumn{2}{|c|}{ SI } & \multicolumn{2}{|c|}{ NO } & \multicolumn{2}{|c|}{ TOTAL } & & & \\
\hline & $\mathrm{N}^{\circ}$ & $\%$ & $\mathrm{~N}^{\circ}$ & $\%$ & $\mathrm{~N}^{\circ}$ & $\%$ & & & \\
\hline$<32$ o $>36 \mathrm{~cm}$ & 26 & $16.99 \%$ & 6 & $3.92 \%$ & 32 & $20.92 \%$ & & & \\
\hline $32-36 \mathrm{~cm}$. & 72 & $47.06 \%$ & 49 & $32.03 \%$ & 121 & $79.08 \%$ & 2.94907407 & $\begin{array}{c}(1.130- \\
7.895)\end{array}$ & 0.023 \\
\hline TOTAL: & 98 & $64.05 \%$ & 55 & $35.95 \%$ & 153 & $100.00 \%$ & & & \\
\hline
\end{tabular}

Fuente: Historias clínicas del Hospital, 2016.

Elaborado por: El investigador. 
En la Tabla se $\mathrm{N}^{\circ} 1$ se observa una asociación significativa entre episiotomía y perímetro cefálico; con un valor de $\mathrm{p}$ de $<$ o igual a 0.05 . Razón de Prevalencia (RP) es de 2.95 y un Intervalo de Confianza (IC) del 95\% (1.130 - 7.895). De las 98 mujeres que recibieron episiotomía, 26 de recién nacidos $(16.99 \%)$, tienen un perímetro cefálico mayor a 36 centímetros, siendo un factor de riesgo con RP $>1$.

Tabla $\mathbf{N}^{\circ}$ 3. Relación entre la episiotomía y peso del recién nacido.

\begin{tabular}{|c|c|c|c|c|c|c|c|c|c|}
\hline \multirow{3}{*}{$\begin{array}{l}\text { PESO DEL } \\
\text { RECIÉN } \\
\text { NACIDO }\end{array}$} & \multicolumn{6}{|c|}{ EPISIOTOMÍA } & \multirow{3}{*}{$\begin{array}{l}\text { Razón de } \\
\text { Prevalencia }\end{array}$} & \multirow{3}{*}{$\begin{array}{c}\text { Intervalo de } \\
\text { Confianza }\end{array}$} & \multirow{3}{*}{$\begin{array}{l}\text { Valor de } \\
\qquad \mathrm{p}\end{array}$} \\
\hline & \multicolumn{2}{|c|}{ SI } & \multicolumn{2}{|c|}{ NO } & \multicolumn{2}{|c|}{ TOTAL } & & & \\
\hline & $\mathrm{N}^{\circ}$ & $\%$ & $\mathrm{~N}^{\circ}$ & $\%$ & $\mathrm{~N}^{\circ}$ & $\%$ & & & \\
\hline $\begin{array}{c}<2500 \text { o } \\
>4000 \text { grs. }\end{array}$ & 47 & $30.72 \%$ & 17 & $11.11 \%$ & 64 & $41.83 \%$ & & & \\
\hline $\begin{array}{c}2500-4000 \\
\text { grs. }\end{array}$ & 51 & $33.33 \%$ & 38 & $24.84 \%$ & 89 & $58.17 \%$ & 2.0599 & $\begin{array}{c}(1.027- \\
4.131)\end{array}$ & 0.040 \\
\hline TOTAL: & 98 & $64.05 \%$ & 55 & $35.95 \%$ & 153 & $100.00 \%$ & & & \\
\hline
\end{tabular}

Fuente: Historias clínicas del Hospital, 2016.

Elaborado por: El investigador.

En la Tabla $\mathrm{N}^{\circ} 3$ si se observa una relación entre la episiotomía y el peso del recién nacido, con un valor de $\mathrm{p}<0.05$. Razón de Prevalencia (RP) es de 2,0599 y un Intervalo de Confianza (IC) del 95\% (1,027-4,131). De las 98 mujeres que recibieron episiotomía, 47 recién nacidos (30.72\%), tienen peso mayor a 4000 gramos. Por lo observado, el peso del recién nacido resulta un factor de riesgo predisponente para la episiotomía, por ser RP $>1$.

Tabla $N^{\circ}$ 4. Relación entre la episiotomía y edad gestacional de término del recién nacido.

\begin{tabular}{|c|c|c|c|c|c|c|c|c|c|}
\hline \multirow{3}{*}{$\begin{array}{c}\text { EDAD } \\
\text { GESTACIONAL } \\
\text { DE TERMINO }\end{array}$} & \multicolumn{6}{|c|}{ EPISIOTOMÍA } & \multirow{3}{*}{$\begin{array}{l}\text { Razón de } \\
\text { Prevalencia }\end{array}$} & \multirow{3}{*}{$\begin{array}{c}\text { Intervalo de } \\
\text { Confianza }\end{array}$} & \multirow{3}{*}{$\begin{array}{l}\text { Valo } \\
\text { de } p\end{array}$} \\
\hline & \multicolumn{2}{|r|}{ SI } & \multicolumn{2}{|c|}{ NO } & \multicolumn{2}{|c|}{ TOTAL } & & & \\
\hline & $\mathrm{N}^{\circ}$ & $\%$ & $\mathrm{~N}^{\circ}$ & $\%$ & $\mathrm{~N}^{\circ}$ & $\%$ & & & \\
\hline$<37$ ó $>42$ & & & & & & & \multirow{4}{*}{0.55208333} & \multirow{4}{*}{$\begin{array}{c}(0.0760- \\
4.033)\end{array}$} & \multirow{4}{*}{0.553} \\
\hline Semanas & 2 & $1.31 \%$ & 2 & $1.31 \%$ & 150 & $2.61 \%$ & & & \\
\hline 37 - 42 Semanas & 96 & $62.75 \%$ & 53 & $34.64 \%$ & 3 & $97.39 \%$ & & & \\
\hline TOTAL: & 98 & $64.05 \%$ & 55 & $35.95 \%$ & 153 & $100.00 \%$ & & & \\
\hline
\end{tabular}

Fuente: Historias clínicas del Hospital, 2016

Elaborado por: El investigador 
Así mismo, al analizar la Tabla $\mathrm{N}^{\circ} 4$ se evidencia que si bien existe relación entre episiotomía $y$ edad gestacional de término con un valor de $p>0.05$. Sin embargo la Razón de Prevalencia (RP) es inferior a la unidad (0.552083), en cuyo Intervalo de Confianza (IC) del 95\% (0,0760 - 4.033) incluye la unidad; por lo tanto, el valor encontrado no puede ser factor de riesgo y factor de protección al mismo tiempo.

Tabla $N^{\circ}$ 5. Relación entre la episiotomía y edad cumplida de la madre al momento del parto.

\begin{tabular}{|c|c|c|c|c|c|c|c|c|c|}
\hline \multirow{3}{*}{$\begin{array}{c}\text { EDAD } \\
\text { CUMPLIDA } \\
\text { DE LA } \\
\text { MADRE }\end{array}$} & \multicolumn{6}{|c|}{ EPISIOTOMÍA } & \multirow{3}{*}{$\begin{array}{c}\text { Razón de } \\
\text { Prevalencia }\end{array}$} & \multirow{3}{*}{$\begin{array}{l}\text { Intervalo de } \\
\text { Confianza }\end{array}$} & \multirow{3}{*}{$\begin{array}{l}\text { Valor } \\
\text { de } p\end{array}$} \\
\hline & \multicolumn{2}{|c|}{ SI } & \multicolumn{2}{|c|}{ NO } & \multicolumn{2}{|c|}{ TOTAL } & & & \\
\hline & $\mathrm{N}^{\circ}$ & $\%$ & $\mathrm{~N}^{\circ}$ & $\%$ & $\mathrm{~N}^{\circ}$ & $\%$ & & & \\
\hline $\begin{array}{c}<18 \text { o }>35 \\
\text { años }\end{array}$ & 35 & $22.88 \%$ & 10 & $6.54 \%$ & 70 & $29.41 \%$ & \multirow{3}{*}{2.500} & \multirow{3}{*}{$\begin{array}{c}(1.123- \\
5.565)\end{array}$} & \multirow{3}{*}{0.022} \\
\hline $18-35$ años & 63 & $41.18 \%$ & 45 & $29.41 \%$ & 83 & $70.59 \%$ & & & \\
\hline TOTAL: & 98 & $64.05 \%$ & 55 & $35.95 \%$ & 153 & $100.00 \%$ & & & \\
\hline
\end{tabular}

Fuente: Historias clínicas del Hospital, 2016

Elaborado por: El investigador

De las 98 mujeres que recibieron episiotomía, el 22.18\% tienen una edad $<18$ años, En la Tabla $\mathrm{N}^{\mathrm{a}} 5$ se aprecia la asociación entre la episiotomía y edad cumplida de la madre con un valor $\mathrm{r}$ de $\mathrm{p}<0.05$ Razón de Prevalencia (RP) es de 2,50 con un Intervalo de Confianza (IC) del 95\% (1.123 - 5.565). Por lo tanto, es un factor de protección.

Tabla $\mathbf{N}^{\circ}$ 6. Relación entre la episiotomía y posición de la madre al momento del parto.

\begin{tabular}{|c|c|c|c|c|c|c|c|c|c|}
\hline \multirow{3}{*}{$\begin{array}{l}\text { POSICIÓN DE } \\
\text { LA MADRE }\end{array}$} & \multicolumn{6}{|c|}{ EPISIOTOMÍA } & \multirow{3}{*}{$\begin{array}{l}\text { Razón de } \\
\text { Prevalencia }\end{array}$} & \multirow{3}{*}{$\begin{array}{c}\text { Intervalo de } \\
\text { Confianza }\end{array}$} & \multirow{3}{*}{$\begin{array}{l}\text { Valor } \\
\text { de p }\end{array}$} \\
\hline & \multicolumn{2}{|c|}{ SI } & \multicolumn{2}{|c|}{ NO } & \multicolumn{2}{|c|}{ TOTAL } & & & \\
\hline & $\mathrm{N}^{\circ}$ & $\%$ & $\mathrm{~N}^{\circ}$ & $\%$ & $\mathrm{~N}^{\circ}$ & $\%$ & & & \\
\hline Litotómica & 98 & $64.05 \%$ & 45 & $29.41 \%$ & 143 & $93.46 \%$ & \multirow{3}{*}{0.315} & \multirow{3}{*}{$\begin{array}{c}(0.246- \\
0.401)\end{array}$} & \multirow{3}{*}{0.000} \\
\hline Vertical & 0 & $0.00 \%$ & 10 & $6.54 \%$ & 10 & $6.54 \%$ & & & \\
\hline TOTAL: & 98 & $64.05 \%$ & 55 & $35.95 \%$ & 153 & $100.00 \%$ & & & \\
\hline
\end{tabular}

Fuente: Historias clínicas del Hospital, 2016

Elaborado por: El investigador

De las 98 mujeres que recibieron episiotomía, el 48.37\% optaron la posición litotómica, pero el $15.69 \%$ optaron por la posición vertical. En la Tabla $\mathrm{N}^{\mathrm{a}} 6$ se aprecia la asociación entre las variables episiotomía y posición materna al momento del parto. Con un valor de $\mathrm{p}$ 
$<0.05$, Razón de Prevalencia (RP) es de 0.315 con un Intervalo de Confianza (IC) del 95\% (0.246 - 0.401). Por lo tanto la posición de la madre es un factor de protección.

\section{Discusión.}

Como cualquier acto quirúrgico, la episiotomía es también responsable de algunas complicaciones asociadas, cuyos beneficios tampoco gozan de evidencia científica que lo apoyen, razón por la cual su práctica rutinaria tiene controversias a pesar de ser comúnmente aplicada en las casas de salud nacionales, regionales y locales.

En Ecuador, en la provincia del Pichincha, de acuerdo a los datos estadísticos publicados por el centro obstétrico del Hospital Isidro Ayora de la ciudad de Quito para el año de 2007 el porcentaje de episiotomías en primigestas llega al 42,6\%, de un total de 7.459 partos vaginales atendidos, con 3.180 intervenciones de episiotomía medio laterales (3). En la provincia de Napo, en una investigación retrospectiva transversal realizada por Zamora L. (4) en el año 2010, en una muestra de 123 mujeres nulíparas, encuentra una tasa de episiotomías del $38 \%$, de las cuales las 47 mujeres son primigestas.

En la provincia del Azuay, la prevalencia de episiotomía en mujeres que acudieron al Hospital Vicente Corral Moscoso en el año 2014 fue del 35,5\%, según estudio realizado en una muestra de 580 mujeres primigestas. (5) En la provincia de Cotopaxi, según la investigación realizada en el Hospital Provincial General de Latacunga, en el año 2015, en una muestra de 137 mujeres primigestas, la prevalencia de episiotomía llega al 62\%, (6).

En esta investigación se encontró que el número de partos eutócicos o normales fueron de 453 , de los cuales $153(33,77 \%)$ corresponden a mujeres nulíparas y $300(66,22 \%)$ a mujeres multíparas. De la muestra de 153 mujeres primigestas, $98(64,05 \%)$ son sometidas a episiotomía, valor que representa la prevalencia de esta práctica, para el año 2016

Como podemos observar, si bien la frecuencia encontrada en investigaciones tiende a ser levemente superior a los valores obtenidos en el país desde hace diez años atrás, indicando por supuesto, que la práctica de la episiotomía no ha disminuyendo desde la entrada en vigencia el componente normativo neonatal del Ministerio de Salud del Ecuador, 2008 (2, 7); y, siendo al mismo tiempo un valor de prevalencia superior al recomendado por la Organización Mundial de la Salud, que oscila entre el 15 al 30\% (1); sin embargo este valor es significativamente inferior a los encontrados a nivel mundial, incluso en países desarrollados cuya porcentaje de prevalencia en episiotomías rutinarias y programadas en primigestas es superior al 80\%, 93\%, Azón L, 2013 (8); 83,7\%, Marques S, 2012 (9).

En la investigación se observa que el $47.08 \%$ de neonatos poseen un perímetro cefálico que se encuentra en un rango comprendido entre 32 y 36 centímetros, con una media más frecuente de 34 centímetros y el $16.99 \%$ tiene un perímetro cefálico superior a los 36 centímetros, reflejada en una razón de prevalencia, así mismo, superior a la unidad (2.94), considerándose estadísticamente como factor de riesgo para el uso de esta técnica obstétrica. Karaçam et al., en un estudio en primigestas en Turquía (10) indica que el mayor tamaño en 
el perímetro cefálico del bebé aumentó la probabilidad de episiotomía (OR, 1,29; IC del 95\%, $1,08-1,53)$

En cuanto al peso de los recién nacidos, 51 neonatos $(33.33 \%)$ cuyas madrea han sido sometidas a episiotomía nacen con peso normal, que según la OMS (1), oscilando entre 2.500 a 4.000 gramos, con una media de 3.250 gramos y 47 neonatos $(30.72 \%)$ nacen con un peso superior a los 4.000 gramos, casos en los cuales se justifica el uso de la episiotomía para facilitar la expulsión del niño. Si el peso promedio de 3.201,75 gramos obtenido de los niños cuyas madres recibieron episiotomía es levemente superior al encontrado por Marques et al. (9), quienes indican que el peso promedio de los recién nacidos a cuyas madres se realizó episiotomía era de 3.110,65 gramos; y, si al mismo tiempo se contrasta el peso promedio de 1.633,93 gramos de los recién nacidos con madre si episiotomía, con valor medio normal de 3.250 gramos y el valor máximo de 4.000 gramos de peso, la mayoría de las episiotomías realizadas en el hospital están justificadas por Karaçam el al. (10) quienes afirman que los pesos medios de los recién nacidos de gestantes con episiotomía fueron significativamente mayores que las embarazadas sin episiotomía. Resultados que coinciden con los obtenidos por Okeke TC, et al. (11), Wu L, et al. (12) y Koskas M, et al. (13) al relacionar peso fetal con incremento de episiotomía.

Las edades gestacionales mayores de 42 semanas no constituyen un factor de riesgo asociado a la episiotomía $(\mathrm{RP}=0.552083)$, las gestaciones posteriores comprenden el $1.3 \%$ de los partos investigados, de las 98 mujeres que recibieron episiotomía, el $62.75 \%$ se encuentran en el rango de las 37 a 42 semanas gestacionales. E1 35,64\% de las mujeres primigestas no reciben episiotomía, que Karaçam el al. (10), se justifica por la tendencia a reducir los partos pos término y finalizar mediante instrumentación.

El mayor porcentaje (41.18\%) de mujeres primigestas que han sido sometidas a episiotomía se encuentra entre los 18 a 35 años, mientras que $22.88 \%$ tenían una edad superior a los 35 años, cuya razón de prevalencia $(\mathrm{RP}=2.5)$ mayor a la unidad nos indica que es un factor de riesgo para la episiotomía y que existe relación y significancia estadística entre los datos ( $\mathrm{p}$ $=0.022$, menor a 0.05), Conclusiones que son corroboradas por Medeiros de Carvalho (14), el que afirma la existencia de $74 \%$ de riesgo de episiotomías en adolescentes, edad que según la OMS puede extenderse hasta los 19 años (1). Por otra parte, Melo Júnior et al. (15) concuerdan en su estudio que el 29,4\% de las pacientes hasta los 22 años mostraron un riesgo mayor de recibir episiotomía. Contrastando con Molina et al. (16) quienes indican que un menor porcentaje de las mujeres primíparas menores de 20 años (36.9\%) recibieron episiotomía, en comparación con edades mayores $(63,1 \%)$. De la misma manera Hernández et al. (17) observaron en primigestas un incremento en los porcentajes de episiotomía a medida que aumentaba la edad materna, 52,38\% en mujeres de 15 a 19 años, y 77,27\% en mayores de 40 años. Ballestero C. et. al. (18) en su investigación concluye que la edad de la madre superior a los 35 años se asocia con una menor incidencia de episiotomía $(45,5 \%)$ frente a edades inferiores $(54,4 \%)$. Los estudios revisados sugieren que el uso de la 
episiotomía no se ha asociado con la edad materna (19). Tal vez esta diferencia se deba a la amplitud de la edad para la maternidad, ya que los estudios comprenden una población de 14-53 años, siendo el 25\% de la muestra mayor de 35 años

El mayor porcentaje de mujeres que recibieron episiotomía $(48.37 \%)$ optaron la posición litotómica, pero el $15.69 \%$ optaron por la posición vertical. Analizando los datos obtenidos se observa que la Razón de Prevalencia (RP) es de 2.0555, mayor a la unidad, determinándose que es un factor de riesgo de la episiotomía y el valor de $\mathrm{p}$ de $0,045, \mathrm{p}<0.05$, indica que existiendo relación entre las variables episiotomía y posición materna al momento del parto. Datos que tienen significancia estadística, cuyo intervalo de confianza no incluye la unidad. Estos resultados coinciden con Ballesteros et al. (24), manifestando que la posición materna durante la fase expulsiva también afecta a la realización de episiotomía y que se debería permitir a las mujeres tener la opción de dar a luz en cualquier posición que se encuentren más cómodas.

\section{Conclusiones .}

- La frecuencia de episiotomía en mujeres primigestas que acudieron al Hospital Luis F Martínez en el año 2016 (64.05\%), es mayor a lo recomendado Organización Mundial de la Salud (16- 30\%). La episiotomía no es un procedimiento aislado e independiente del resto de las prácticas obstétricas, sino que está asociado a la práctica de determinadas variables clínicas que influyen en el aumento de la tasa de este procedimiento. La edad predominantes es entre 18 y 35 años El estado civil es Unión Libre. La mayoría han llegado a una instrucción Básica y Bachillerato y un número mínimo de pacientes analfabetas.

- Las primigestas entre los 18 y 35 años de edad tuvieron 2,5 veces más posibilidades estadísticas de recibir episiotomía que aquellas cuya edad era $<18$ y $>35$ años, con un valor de $\mathrm{p}$ de 0,022 . Las mujeres con neonatos con un peso entre los 2500 y 4000 gramos en este estudio obtuvieron 2,05 veces menos posibilidades estadísticas de recibir episiotomías que aquellas con neonatos cuyo peso fue $<2500$ y $>4000$ gramos, con un valor de $\mathrm{p}$ de 0,040 . Las mujeres con recién nacidos cuyo perímetro cefálico se encontraba entre 32 y $36 \mathrm{~cm}$ tuvieron 2,94 veces menos posibilidades estadísticas de recibir episiotomía que aquellas con neonatos cuyo perímetro cefálico fue de $<32$ y $>36 \mathrm{~cm}$, con un valor de p 0,023. En cuanto a la de edad gestacional a término del recién nacido y la realización de la episiotomía no se encontró una relación estadísticamente significativa. En relación a la posición materna durante el parto se encontró una asociación entre esta variable y la episiotomía siendo la posición litotómica la más frecuente con un valor de $\mathrm{p}$ de 0,00 . Con un valor de $\mathrm{RP}<$ 1 se trata de un factor de protección y no de riesgo. 
- Sobre la base de los resultados obtenidos se comprueba la hipótesis de investigación: la prevalencia de episiotomías en pacientes primigestas asociados con perímetro cefálico fetal, peso y talla del neonato, edad gestacional de término, edad, posición al momento del parto y edad gestacional; que acudieron al Hospital Luis F. Martínez de la ciudad de Cañar, NO ha disminuido con la aplicación de la normativa materno neonatal del Ministerio de Salud Pública. Si deseamos disminuir la tasa de episiotomía, será necesario tener en cuenta los factores que condicionan su práctica y establecer políticas de reducción del este procedimiento, consensuado con todos los profesionales sanitarios que asisten a la mujer en su proceso de parto.

\section{Referencias bibliográficas.}

Organización Mundial de la Salud. Cuidados en el parto normal: una guía práctica [Internet]. Ginebra: Organización Mundial de la Salud; Informe técnico del Departamento de Investigación y Salud Reproductiva.

Disponible en: http://whqlibdoc.who.int/hq/1996/WHO_FRH_MSM_96.24_spa.pdf

Ministerio de Salud Pública Ecuatoriano. MSP. Componente Normativo Materno. 2008.

Disponible en: http://www.maternoinfantil.org/archivos/smi_D64.pdf

Hospital Gineco Obstétrico Isidro Ayora. Página Web. 2013.

Disponible en:

http://www.hgoia.gob.ec/documentos/pdf/estadisticas/informe2007/CIRUGIAS\%20POR\% 20SERVICIOS.pdf .

Molina C, Ortiz M, Huete M, et al. Análisis de la implantación de una política de episiotomía selectiva en un hospital comarcal. Evidentia. 2010 ene-mar; 6(25). España.

Disponible en:

http://www.google.com.ec/url?sa=t\&rct=j\&q=\&esrc=s\&source=web\&cd=20\&ved=0CGoQ FjAJOAo\&url=http\%3A\%2F\%2Fxa.yimg.com\%2Fkq\%2Fgroups\%2F27500701\%2F18059 98118\%2Fname $\% 2 \mathrm{FAn} \% 25 \mathrm{C} 3 \% 25 \mathrm{~A} 1$ lisis\%2Bde $\% 2 \mathrm{Bla} \% 2 \mathrm{Bimplantaci} \% 25 \mathrm{C} 3 \% 25 \mathrm{~B} 3 \mathrm{n} \%$ 2Bde\%2Buna\%2Bpol\%25C3\%25ADtica\%2Bde\%2Bepisiotom\%25C3\%25ADa\%2Bselecti va\%2Ben\%2Bun\%2Bhospital\%2Bcomarcal.doc\&ei=dthRU87PMujLsQSx4C4Dw\&usg=A FQjCNFiNvVPNRT1sJTs0gHY48M3jhb3Ew\&bvm=bv.6505 8239, d.cWc 
Gualán C, Guanoluisa O. Prevalencia de episiotomía y factores asociados, en pacientes del centro obstétrico del Hospital Vicente Corral Moscoso, Cuenca-Ecuador 2014. Trabajo de pregrado previo a la obtención de Título De Médico General. 2015

Zamora C, Lady G. La episiotomía: ventajas y desventajas del Uso y no uso en pacientes de parto normal del Hospital José María Velasco Ibarra, Tena, enero- agosto 2010.

Ministerio de Salud Pública Ecuatoriano. Control prenatal guía de Práctica Clínica 2015 MSP Ecuador, DNN MSP

Disponible en: http://es.slideshare.net/pomicin/gpc-control-prenatal-2015-msp-ecuador2015

Azon L, Garcia M, Subiron V. Análisis de los resultados obstétricos y neonatales de los partos inducidos en nulíparas en el Hospital Clínico de Zaragoza. Matronas Prof. 2013; 14(2): 52-59. Disponible en:

https://www.google.com.ec/url?sa=t\&rct=j\&q=\&esrc=s\&source=web\&cd=1\&cad=rja\&uac $\mathrm{t}=8 \& \mathrm{ved}=0 \mathrm{CBwQFjAA} \& u r \mathrm{l}=\mathrm{http} \% 3 \mathrm{~A} \% 2 \mathrm{~F} \% 2 \mathrm{Fwww}$.federacionmatronas.org\%2Frs $\% 2 \mathrm{~F} 1$ $043 \% 2 \mathrm{Fd} 112 \mathrm{~d} 6 \mathrm{ad}-54 \mathrm{ec}-438 \mathrm{~b}-9358$ 4483f9e98868\%2F69a\%2Ffd\%2F1\%2Ffilename\%2Foriginalesultadosobstetricos.pdf\&ei= COvQVPGrJcX_gwS1koLYCw\&usg=AFQjCNFKoqRgeJ mleNHfyWvvanqaZykonQ

Marques S, Fleury L, Machado S, et al. Práctica de la episiotomía y factores maternos y neonatales relacionados.2012oct/dec;14(4):779-85. Brasil.

Disponible en: http://www.fen.ufg.br/fen_revista/v14/n4/pdf/v14n4a05.pdf

Karaçam Z, Ekmen H, Çalışır H, Şeker S, Prevalencia de la episiotomía en primíparas, relacionando condiciones y efectos de la episiotomía en los materiales de sutura usada, dolor perineal, la cicatrización de heridas 3 semanas después del parto, en Turquía: Un estudio de seguimiento prospectivo. Diario iraní de Enfermería y Obstetricia de Investigación | MayoJunio de 2013 | Vol. 18 | Número 3.

http://www.cochrane.org/es/CD008977/resutura-de-las-heridas-perineales-el-area-entre-lavagina-y-el-ano-por-dehiscencia-en-comparacion

Okeke T, Uqwu E, Okezie O, Enwereji J, Ezenyeaku C, Ikeako L. Trends and determinants of episiotomy at the University of Nigeria Teaching Hospital, Enugu, Nigeria. Niger J Med. 2012 Jul-Sep;21(3):304-7. Pubmed PMID:23304925.

http://www.redalyc.org/pdf/3658/365834854022_2.pdf

Wu L, Malhotra R, Allen J Jr, Lie D, Tan T, Ostbye T. Risk factors and midwife-reported reasons for episiotomy in women undergoing normal vaginal delivery. Arch Gynecol Obstet. 2013 Dic; 288(6):1249-56

Disponible en: https://www.ncbi.nlm.nih.gov/pubmed/23708390 
Koskas M, Caillod A, Fauconnier A, Bader T. Maternal and neonatal consequences induced by the French recommendations for episiotomy practice. Monocentric study by 5409 vaginal deliveries. Gynecol Obstet Fertil.2009 Sep;37(9):697-702.

Carvalho C, Coelho M, Moraes F, Prevalence and factors associated with practice of episiotomy at a maternity school in Recife, Pernambuco, Brazil. Rev. Assoc. Med. Bras. [Internet]. 2010 [cited 2016 Sep 30]; 56(3): 333-339. Available from: http://www.scielo.br/scielo.php?script=sci_arttext\&pid=S010442302010000300020\&lng=en. http://dx.doi.org/10.1590/S0104-42302010000300020

Melo L, Freire S. Factores asociados con la práctica de episiotomía en un hospital universitario. Brasil. Rev. Cienc Med, Campinas, 15(2):95-101, mar./abr., 2006. Disponible en:

http://periodicos.puccampinas.edu.br/seer/index.php/cienciasmedicas/article/viewFile/1119/ 1094

Molina C, Ortiz M, Huete M, et al. Análisis de la implantación de una política de episiotomía selectiva en un hospital comarcal. Evidentia. 2010 ene-mar; 6(25). España.

Disponible

en: http://www.google.com.ec/url?sa=t\&rct=j\&q=\&esrc=s\&source=web\&cd=20\&ved=0CGoQ FjAJOAo\&url=http\%3A\%2F\%2Fxa.yimg.com\%2Fkq\%2Fgroups $\% 2 F 27500701 \% 2 F 18059$ 98118\%2Fname $\% 2$ FAn $\% 25 \mathrm{C} 3 \% 25 \mathrm{~A} 11$ isis $\% 2 \mathrm{Bde} \% 2 \mathrm{Bla} \% 2 \mathrm{Bimplantaci} \% 25 \mathrm{C} 3 \% 25 \mathrm{~B} 3 \mathrm{n} \%$ 2Bde\%2Buna\%2Bpol\%25C3\%25ADtica\%2Bde\%2Bepisiotom\%25C3\%25ADa\%2Bselecti va\%2Ben\%2Bun\%2Bhospital\%2Bcomarcal.doc\&ei=dthRU87PMujLsQSx4C4Dw\&usg=A FQjCNFiNvVPNRT1sJTs0gHY48M3jhb3Ew\&bvm=bv.6505 8239,d.cWc

Hernández J, Azón E, Mir E, Peinado R, Val B, Mérida A. Factores que influyen en la realización de una episiotomía selectiva en mujeres nulíparas. Enferm. glob. [Internet]. 2014 Jul [citado 2016 Ago 19]; 13(35): 398-411. Disponible en: http://scielo.isciii.es/scielo.php?script=sci_arttext\&pid=S1695$61412014000300022 \& \operatorname{lng}=\mathrm{es}$

Ballesteros C., Martienz M., Aseguer M., Carrillo C., Canteras M. Episiotomía en el marco de la atención al parto normal. AMRM. Asociación de matronas de la región de Murcia. España. 2014.

Consultado el 13 de mayo del 2016

Disponible en:

https://www.google.com/search?sclient=psy-ab\&client=firefox-bab\&biw $=1366 \& b i h=636 \&$ noj $=1 \& q=$ episiotomia,+ Es + considerada + como + una + de + las + ope raciones $+\mathrm{m} \% \mathrm{C} 3 \% \mathrm{~A} 1 \mathrm{~s}+$ comunes + realizadas $+\mathrm{en}+$ mujeres ++ primigestas $\&$ oq $=$ episiotomia,+ Es + considerada + como + una + de + las + operaciones $+\mathrm{m} \% \mathrm{C} 3 \% \mathrm{~A} 1 \mathrm{~s}+$ comunes + realizadas + en + 
mujeres++primigestas\&gs_l=serp.3...51404.55142.1.55744.13.13.0.0.0.0.332.1528.2-

$5 \mathrm{j} 1.6 .0 \ldots . .0 \ldots 1.1 .64 . \operatorname{serp} .7 .4 .1039 \ldots 30 \mathrm{i} 10 \mathrm{k} 1$. TeLXJa7LUM0\&gfe_rd=cr\&ei $=\mathrm{cxdjWdNx} 1 \mathrm{Y}$ mEBMvShyA

Gibbs K, Haney N, Obstetricia y Ginecología de Danforth, 10 ma Edición, España, ed. 2009, págs. 31-32-33-34

Rouviere H, Delmas A, Anatomía Tomo 2, Anatomía topográfica del periné, 11va Edición, ed. 2005, págs. 680-681-682

Shuarcz R, Fescina R, Duverges C, Obstetricia, 6ta Edición, ed. 2005, págs. 485-486-487

Cunningham L, Bloom H, Wiliams Obstetricia, Spong,23va Edicion, ed. 2011 pàgs 401402-403

Botero J, Henao G, Jubiz A, Obstetricia y Ginecología, 9na Edición Colombia, ed. 2015, pág. 148 


\section{Para citar el artículo indexado.}

Moncayo C. \& Vázquez V. . (2018). Frecuencia y factores asociados a episiotomía en primigestas atendidas en el hospital "Luis f. Martínez", año 2016. Revista electrónica Ciencia Digital 2(3), 257-272. Recuperado desde: http://cienciadigital.org/revistacienciadigital2/index.php/CienciaDigital/article/view/151/13 $\underline{6}$

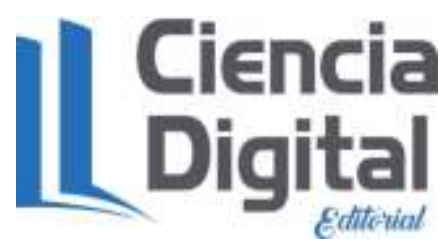

El artículo que se publica es de exclusiva responsabilidad de los autores y no necesariamente reflejan el pensamiento de la Revista Ciencia Digital.

El articulo queda en propiedad de la revista y, por tanto, su publicación parcial y/o total en otro medio tiene que ser autorizado por el director de la Revista Ciencia Digital.
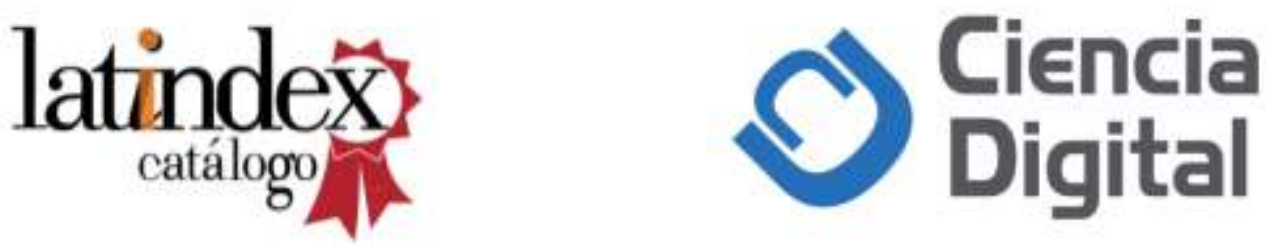